\title{
MIOTICS
}

THE commonly used miotics are parasympathomimetic drugs which imitate or potentiate the action of acetylcholine by producing contraction of the sphincter pupillae and ciliary muscles. The two main groups of these drugs are:

(1) Cholinergic drugs, which have an action similar to acetylcholine (i.e. pilocarpine, carbachol and methacholine);

(2) Anticholinesterases, which inhibit or destroy cholinesterase, thus causing a prolonged accumulation of naturally produced acetylcholine.

\section{Uses of Miotics}

(1) Closed-angle Glaucoma.-Contraction of the sphincter pupillae causes the iris diaphragm to become taut, thus preventing both crowding of the angle of the anterior chamber by the peripheral iris and relaxation of the peripheral iris which predisposes to iris bombé. Cholinergic drugs are valuable in the short-term treatment of closed-angle glaucoma; anticholinesterase drugs are contraindicated in this condition as they tend to produce pupillary block.

(2) Simple Glaucoma.-Miotics cause an increase in the outflow of aqueous from the anterior chamber, the exact mechanism of which is unknown, although it is believed that contraction of the ciliary muscle may cause a slight backward movement of the scleral spur. This in turn may exert a pull on the trabecular meshwork thus increasing its permeability.

(3) Accommodative Esotropia.-The long-acting anticholinesterase drugs, D.F.P., echothiophate, and demecarium bromide, are valuable in the treatment of certain cases of accommodative squint, whether associated with excessive hypermetropia or with an abnormally high AC/A ratio. They act by stimulating accommodation peripherally thus reducing the excessive stimulus to convergence.

\section{Cholinergic Drugs}

(1) Acetylcholine.-Produces a marked miosis lasting 20 minutes or more when directly introduced into the anterior chamber. This effect is partially opposed by a previous retrobulbar or subconjunctival injection of adrenaline, but is enhanced by the simultaneous instillation of pilocarpine or physostigmine. It has little effect when instilled into the conjunctival sac.

Indications: (a) In anterior segment surgery when miosis is required (cataract extraction, penetrating keratoplasty, glaucoma surgery). (b) To counteract the effects of atropine.

Used as a 1:100 solution.

(2) Methacholine (Mecholyl).-A short-acting synthetic cholinergic drug which penetrates the cornea poorly. Contraindicated in patients with asthma. Systemic reactions frequently occur.

Indications: (a) As a diagnostic test for Adie's pupil; a 2.5 per cent. solution causes a miosis on the affected side. (b) In simple glaucoma where it reduces the ocular tension, although its longterm use is contraindicated because of its vasodilatory effect.

Used in concentrations of 2.5 to 20 per cent.

(3) Carbachol (Doryl).-A synthetic cholinergic drug, more potent and longer-acting than pilocarpine, although it does not penetrate the cornea as well except in the presence of a corneal abrasion when its use is contraindicated to prevent excessive penetration and side-effects.

Indications: Simple glaucoma, where it is a useful alternative to pilocarpine and to the other miotics where resistance or intolerance have developed.

Used in concentrations of $0 \cdot 75,1 \cdot 5$, and 3 per cent., 4 to 8 -hourly.

(4) Pilocarpine.-The most widely-used miotic, producing a miosis in 10 to 15 minutes which lasts several hours. Unlike some other cholinergic drugs its vasodilatory effect is not marked.

Indications: (a) Primary glaucoma. (b) To reverse the effects of short-acting mydriatics.

Used in concentrations of 0.5 to 4 per cent. As its effects last 6 to 8 hours, it should be used at least three times a day in the treatment of simple glaucoma, although in acute closed-angle glaucoma it may be administered as frequently as once a minute. 


\section{Anticholinesterase Drugs}

(1) Physostigmine (Eserine). - Causes a rapid miosis which is maximal in 30 minutes and lasts 24 to 72 hours.

Indications: (a) Simple glaucoma. Its prolonged use may result in allergic manifestations and in the formation of iris cysts. (b) Closed-angle glaucoma. (c) To counteract mydriasis, except when the production of acetylcholine is reduced, for example following retrobulbar anaesthesia.

Used in concentrations of $\mathbf{0 . 2 5}$ to 1 per cent., two or three times a day. It is oxidized in the presence of light and air when it turns pink and loses its potency.

(2) Neostigmine (Prostigmine)._-A synthetic anticholinesterase drug, more stable but less powerful than physostigmine, but with fewer side-effects.

Indications: Simple glaucoma, in patients who have become sensitive to other miotics.

Used in concentrations of 3 to 5 per cent., 4 to 6-hourly.

(3) Echothiophate (Phospholine).-A long-acting anticholinesterase inhibitor, producing maximal miosis in 1 hour and lasting several days.

Indications: (a) Simple glaucoma. (b) Aphakic glaucoma. (c) Accommodative squint.

Used in concentrations of 0.06 to 0.25 per cent., once or twice a day. It must be kept refrigerated.

(4) Demecarium bromide (Humorsol). - A long-acting anticholinesterase similar in action and indications to echothiophate. It may, however, be kept at room temperature.

Used in concentrations of 0.125 and 0.25 per cent. once or twice a day.

(5) DFP (Dyflos, Floropryl).-A potent and relatively irreversible cholinesterase inhibitor with similar actions and indications to echothiophate and demecarium bromide. If it comes into contact with tears it is rapidly hydrolyzed and inactivated.

Used in concentrations of 0.01 to 0.1 per cent. once or twice a day.

Ocular

Side-Effects

(1) Impairment of Vision.-This is due to miosis and is increased by the presence of lens opacities. Miosis may produce a general depression of the visual field and increase the size of field defects.

(2) Accommodative Effects.-Ciliary spasm tends to produce a temporary myopia, most marked with the long-acting anticholinesterase drugs, which may require the use of a myopic correction.

(3) Iris Cysts.-The prolonged topical administrations of miotics, particularly the long-acting anticholinesterases, may result in the formation of cysts along the pupillary border. Their occurrence may be reduced by the simultaneous administration of adrenaline ( 1 to 2 per cent.).

(4) Pain and Headache.-Due to ciliary spasm, usually temporary and relieved by salicylates.

(5) Anterior Uveitis.-A faint flare is commonly seen after the prolonged use of miotics, and posterior synechiae may be formed, particularly when the long-acting anticholinesterases are used.

(6) Conjunctival Irritation.-Common with physostigmine, the long-continued use of which may lead to the development of a chronic follicular conjunctivitis and contact dermatitis.

(7) Detachment of the Retina.-This may occur when the potent anticholinesterases are used and these drugs should therefore be avoided in a patient with a history of a retinal detachment.

(8) Closed-angle Glaucoma.-The anticholinesterase drugs are contraindicated in patients with narrow angles in whom an attack of angle closure may be precipitated.

(9) Lens Opacities.-Anterior subcapsular opacities have been reported in a high percentage of patients on the stronger anticholinesterases, particularly after treatment for several months, and particularly in the older age groups.

\section{Systemic}

These occur particularly with the long-acting anticholinesterases and are the result of stimulation of the parasympathetic nervous system. Nausea, vomiting, abdominal cramps, diarrhoea, bronchospasm, bradycardia, increased sweating and salivation, muscular cramps, anxiety, tremor, and tension headaches may all occur. These side-effects are usually mild and disappear when the drug is discontinued. Severe symptoms may be treated with systemic atropine or pralidoxime (PAM). 